\title{
Hepatotoxicity due to tocilizumab and anakinra in rheumatoid arthritis: two case reports
}

This article was published in the following Dove Press journal:

International Journal of General Medicine

8 September 2011

Number of times this article has been viewed

\section{Mahmud Mahamid ${ }^{1,3}$ \\ Kalman Paz \\ Mader Reuven ${ }^{4}$ \\ Rifaat Safadi ${ }^{1,2}$}

'Liver Unit, Holy Family Hospital, Nazareth, Israel; ${ }^{2}$ Hadassah Medical Center, ${ }^{3}$ Shaare Zedek Medical Center, Jerusalem, Israel; ${ }^{4}$ Rheumatic Diseases Unit, Ha'Emek Medical Center, Afula, Israel
Correspondence: Mahmud Mahamid Digestive Diseases Institute, Shaare Zedek Medical Center, 12 Bayit Street, Jerusalem, Israel 91031

Tel +97226666064

Fax+972 26666050

Email mmahamid@szmc.org.il
Abstract: Elevation of liver enzymes in patients with rheumatoid arthritis treated with the biological agents, tocilizumab and anakinra, is now well documented. However, histological characterization of these effects and outcomes has not been defined. Here we report toxic liver effects in two women with rheumatoid arthritis, refractory to all nonbiological therapies, following treatment with anakinra and tocilizumab. Liver biopsy in both cases showed focal necrosis of hepatocytes as a hallmark of drug toxicity, along with steatosis and early fibrosis. In addition, the patient treated with anakinra demonstrated inflammatory changes. Tocilizumab was continued with no further deterioration in liver function. Withdrawal of anakinra led to rapid normalization of liver function. The biological agents, tocilizumab and anakinra, may result in significant histological hepatic changes, including necrosis, but despite this, the outcome appears to be good.

Keywords: tocilizumab, anakinra, interleukin receptors, lymphocytes, liver injury, rheumatoid arthritis

\section{Introduction}

Based on well described efficacy and safety studies, tocilizumab, a recombinant humanized anti-interleukin-6 monoclonal antibody, and anakinra, an interleukin-1 receptor antagonist, have been approved for the treatment of refractory rheumatoid arthritis in adults. ${ }^{1-3}$ Both agents suppress the inflammation and cartilage degradation associated with rheumatoid arthritis, and both have been reported as safe and well tolerated for up to three years of continuous use in patients with the disease. ${ }^{1-4}$ Tocilizumab is given intravenously on a monthly schedule and anakinra is given subcutaneously once a day. ${ }^{4,5}$ Common infusion reactions include headache, nausea, flu-like symptoms, and local injection site reactions. ${ }^{1,2}$ The most severe adverse events are infections (mainly pneumonia and upper respiratory tract infections), sinusitis, and diarrhea. ${ }^{1-4}$ Sepsis and opportunistic infections, including mycobacterial infection and histoplasmosis, have occasionally been reported..$^{1-3}$ In a large study involving 1346 patients treated with anakinra or tocilizumab, a total of 15 fatalities occurred during a three-year period. These were due to cardiac arrest, myocardial infarction, cerebrovascular accident, ventricular fibrillation, sepsis, upper gastrointestinal hemorrhage, malignant melanoma, lymphoma, and suicide. ${ }^{1-4}$

Liver injury in patients treated with tocilizumab and anakinra is now well recognized. ${ }^{1-3,6}$ Increased serum transaminase and bilirubin levels are common, requiring dose adjustments or discontinuation. ${ }^{1,7}$ Liver injury was resolved or alleviated in most cases following reduction of the dose of tocilizumab. ${ }^{7,3}$ In general, 
neither alanine transaminase elevations nor increases in bilirubin are associated with clinical evidence of hepatic decompensation. ${ }^{1,6,7}$ Indeed, other etiologies are suspected in such cases, including autoimmune hepatitis, steroid-induced steatohepatitis, and other drug-induced hepatotoxicity, in particular, methotrexate. ${ }^{1,2,7}$ Although adverse effects of anakinra and tocilizumab on the liver have been reported, histological evaluation of this pathology has not been reported. Here we describe two patients with rheumatoid arthritis who developed hepatotoxicity on tocilizumab and anakinra. We document the histological changes and their good clinical outcomes.

\section{Case report I}

A 46-year-old woman, initially diagnosed as having rheumatoid arthritis in May 2000, was treated with methotrexate $12.5 \mathrm{mg}$ weekly for 10 years. Due to progressive arthralgia and severe morning stiffness, despite lack of bony erosions, treatment with methotrexate was discontinued, and tocilizumab $8 \mathrm{mg} / \mathrm{kg}$ (total dose $640 \mathrm{mg}$ ) once monthly was instituted intravenously in January 2010, with a good clinical response. Before administration of tocilizumab, her white blood cell count was normal, hemoglobin was $14 \mathrm{~g} / \mathrm{dL}$, and platelet count was $178,000 \mathrm{mcL}$. Biochemical data were within the normal range, including all liver and renal function tests.

On February 12, 2010, a few days after the second infusion, the patient complained of extreme general weakness and fatigue. Laboratory examinations at that time remained within normal limits, including liver function tests and hematological parameters. Abdominal ultrasound showed mild fatty liver and mild splenomegaly. Complete virological, metabolic, and autoimmune markers were normal. The patient consumed no alcohol and had no other risk factors, including medications for liver disease. Due to ongoing severe fatigue, weakness, and findings of fatty liver and splenomegaly on initial ultrasound, a liver biopsy was performed in July 2010. Histological examination revealed focal hemorrhagic necrosis of hepatocytes, with areas of macrovesicular steatosis (Figure 1). Technetium staining showed perisinusoidal fibrosis, with no evidence of inflammation or cellular infiltrates. We could not find any evidence of hepatitis or other cause of hepatotoxicity to explain the findings in this patient.

\section{Case report 2}

This case was a 49 -year-old woman with a 12-year history of rheumatoid arthritis, and hypertension treated with beta-blockers, and moderate obesity (body mass index 31 ). She had been treated with methotrexate for five years and

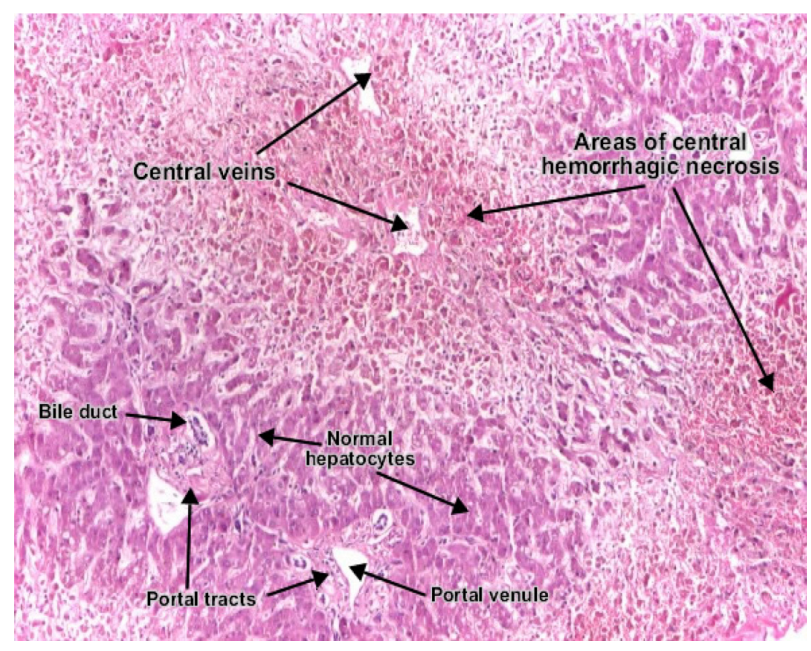

Figure I Areas of central hemorrhagic necrosis in the liver.

intermittent nonsteroidal anti-inflammatory drugs. She had been known to have nonalcoholic fatty liver disease, with consistently normal liver function tests. Other than obesity, she had no other risk factors for chronic liver disease and was taking no other hepatotoxic medications. Due to worsening of her rheumatoid symptoms, in particular morning stiffness and joint pain, anakinra $100 \mathrm{mg}$ /day subcutaneously was instituted in November 2009. Before administration of anakinra, her complete blood count and biochemical data, including liver function tests, were all within the normal range. Two months later, she developed severe fatigue, and laboratory examination at that time revealed elevated liver function tests, with aspartate aminotransferase $30 \mathrm{U} / \mathrm{L}$, alanine aminotransferase $50 \mathrm{U} / \mathrm{L}$, lactate dehydrogenase $584 \mathrm{U} / \mathrm{L}$, alkaline phosphatase $161 \mathrm{U} / \mathrm{L}$, gamma glutamyltranspeptidase $85 \mathrm{U} / \mathrm{L}$, and normal total bilirubin and amylase. Complete blood count, coagulation profile, and urinalysis remained normal. Full virological, autoimmune, and metabolic tests were normal. Ultrasound revealed a fatty liver and mild hepatosplenomegaly. Anakinra was discontinued, fatigue resolved, and liver function tests returned to normal within three weeks.

A liver biopsy (Figure 2) revealed extensive macrovesicular steatosis ( $80 \%$ of the biopsy volume) with focal necrosis of hepatocytes and mild infiltrate of lymphocytes around the areas of necrosis and sinusoids of the liver. Technetium staining showed intraportal fibrosis and perisinusoidal fibrosis. These findings were compatible with those of the regression phase of acute liver injury.

\section{Discussion}

We present here for the first time histological liver changes in two female patients due to biological treatment for 


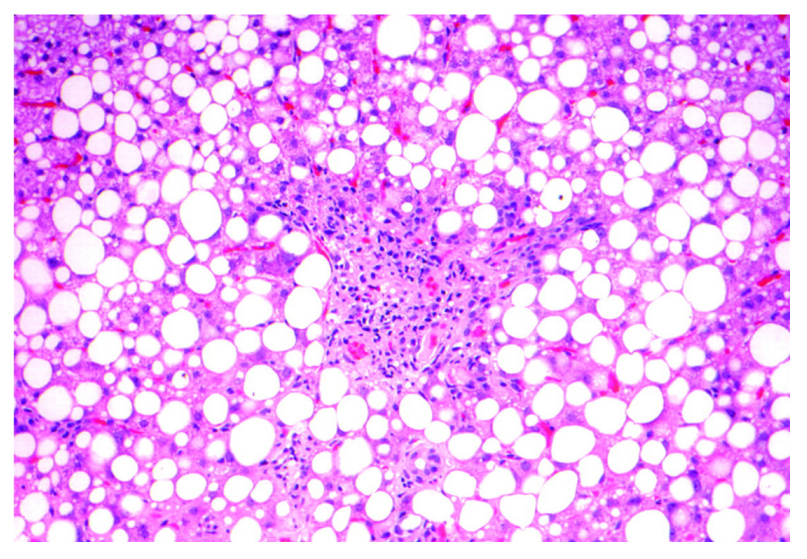

Figure 2 Macrovesicular steatosis in $80 \%$ of the biopsy volume with focal necrosis of hepatocytes and mild infiltrate of lymphocytes around necrotic lesions and on sinusoids of the liver.

rheumatoid arthritis. The common histological changes included focal necrosis with perisinusoidal fibrosis and steatosis. Unique to the first patient treated with tocilizumab was focal hemorrhagic necrosis without cellular infiltrates. The anakinra-treated patient showed steatosis with focal necrosis and lymphocytic infiltrates. She had well documented fatty liver disease, although her liver function tests were always within normal limits. It is reasonable to assume that development of steatosis was induced by tocilizumab in the first patient, and progression from nonalcoholic fatty liver disease to nonalcoholic steatohepatitis was due to anakinra in the second patient. Interesting to note was that despite the remarkable histological findings, the clinical manifestations were nonspecific, and the liver enzymes were normal in the first case and only slightly elevated in the second case.

Although one patient had an increased body mass index and both were exposed to steroid treatment, steatosis and steatohepatitis could also be a manifestation of hepatotoxicity. ${ }^{8}$ The mechanism by which tocilizumab and anakinra is implicated in the pathogenesis of the fatty infiltrate formation is unclear. Leptin, plasminogen activator inhibitor-1, and insulin resistance are suggested to provide an etiopathological link. ${ }^{6,910,11}$ However, more studies are needed to confirm this complex mechanism.

The liver is unique amongst the body organs in its ability to regenerate fully after extensive liver damage. ${ }^{9}$ Hepatocyte activation requires priming by inflammatory cytokines, such as interleukin-1, interleukin-6, and tumor necrosis factor alpha. ${ }^{7,10,11}$ Once primed, the hepatocytes respond to a number of growth factors, including hepatocyte growth factor released by stellate cells. ${ }^{6,12}$ In mice deficient in interleukin-1 and interleukin-6, regeneration of the liver was suppressed after partial hepatectomy. ${ }^{9,8}$ Therefore, interleukin-6 signaling is currently the best characterized pathway thought to be associated with hepatoprotection. ${ }^{10,11}$

Tocilizumab and anakinra are anti-interleukin-6 and anti-interleukin-1 receptors that inhibit interleukin- 6 and interleukin-1 signal transduction, respectively. The pathological findings suggest acute liver injury. ${ }^{6,12}$ One strange phenomenon was the absence of marked elevation of the serum aminotransferase level, suggesting that the hepatocytes had gone into apoptosis rather than necrosis or autophagic cell death, and that hepatic regeneration had been markedly suppressed by blockade of the interleukin- 1 and interleukin- 6 pathways. ${ }^{1,6,7,10,11}$

\section{Conclusion}

Here we describe, for the first time, histological changes in two cases of acute liver injury due to tocilizumab and anakinra, two biological agents commonly used in the treatment of rheumatoid arthritis. Drugs targeting interleukin-6 and interleukin-1 are being widely used for various other autoimmune diseases as well, and could induce severe liver injury by promoting apoptosis and inhibiting liver regeneration, as in our two cases. Physicians need to be aware of the impact of these drugs on the liver.

\section{Disclosure}

The authors report no conflicts of interest in this work.

\section{References}

1. Fleischmann RM, Tesser J, Schiff MH, et al. Safety of extended treatment with anakinra in patients with rheumatoid arthritis. Ann Rheum Dis. 2006;65:1006-1012.

2. Donahue KE, Gartlehner G, Jonas DE, et al. Systemic review: Comparative effectiveness and harms of disease-modifying medications for rheumatoid arthritis. Ann Intern Med. 2008;148:124-134.

3. Mertens M, Singh JA. Anakinra for rheumatoid arthritis. Cochrane Database Syst Rev. 2009;1:CD005121.

4. Weisman MH. What are the risks of biological therapy in RA? An update on safety. J Rheumatol Suppl. 2002;65:33-38.

5. Genovese MC, Cohen S, Moreland L, et al. Combination therapy with etanercept and anakinra in the treatment of patients with rheumatoid arthritis who have been treated unsuccessfully with methotrexate. Arthritis Rheum. 2004;50:1412-1419.

6. Juhan-Vague I, Alessi MC, Mavri A, Morange PE. Plasminogen activator inhibitor-1, inflammation, obesity, insulin resistance and vascular risk. J Thromb Haemost. 2003;1:1575-1579.

7. Cunnane G, Madigan A, Murphy E, FitzGerald O, Bresnihan B. The effect of treatment with interleukin-1 receptor antagonist on the inflamed synovial membrane in rheumatoid arthritis. Rheumatology (Oxford). 2001;40:62-69.

8. Gewiese-Rabsch J, Drucker C, Malchow S, Scheller J, Rose-John S. Role of IL- 6 trans-signaling in $\mathrm{CCl}_{4}$ induced liver damage. Biochim Biophys Acta. 2010;1802:1054-1061.

9. Garcea G, Maddern GJ. Liver failure after major hepatic resection. J Hepatobiliary Pancreat Surg. 2009;16:145-155. 
10. Lam SP, Luk JM, Man K, et al. Activation of interleukin-6 induced glycoprotein 130/signal transducer and activation of transcription 3 pathway in mesenchymal stem cells enhances hepatic differentiation, proliferation, and liver regeneration. Liver Transpl. 2010; 16: $1195-1206$.
11. Cressman DE, Greenbaum LE, DeAngelis RA, et al. Liver failure and defective hepatocyte regeneration in interleukin-6-deficient mice. Science. 1996;247:1379-1383.

12. Mosmann TR, Moor KW. The role of IL-10 in crossregulation of TH1 and TH2 responses. Immunol Today. 1991;12:A49-A53.

\section{Publish your work in this journal}

The International Journal of General Medicine is an international, peer-reviewed open-access journal that focuses on general and internal medicine, pathogenesis, epidemiology, diagnosis, monitoring and treatment protocols. The journal is characterized by the rapid reporting of reviews, original research and clinical studies across all disease areas.
A key focus is the elucidation of disease processes and management protocols resulting in improved outcomes for the patient.The manuscript management system is completely online and includes a very quick and fair peer-review system. Visit http://www.dovepress.com/ testimonials.php to read real quotes from published authors.

Submit your manuscript here: http://www.dovepress.com/international-journal-of-general-medicine-journal 